\title{
The New Virtual Reality: How COVID-19 Will Affect the Gastroenterology and Hepatology Fellowship Match
}

\author{
Niharika Mallepally ${ }^{1} \cdot$ Mohammad Bilal $^{2} \cdot$ Yasmin G. Hernandez-Barco $^{3} \cdot$ Malorie Simons $^{4} \cdot$ Tyler M. Berzin $^{2}$. \\ Amy S. Oxentenko ${ }^{5}$
}

Published online: 1 July 2020

○) Springer Science+Business Media, LLC, part of Springer Nature 2020

\section{Introduction}

The COVID-19 pandemic has seriously disrupted medical training across every specialty worldwide, including gastroenterology (GI) fellowship training [1, 2]. GI fellows have experienced significant reductions in endoscopy procedure volumes [3], cancelation of outpatient clinics, reformatting of lectures to virtual platforms, redeployment to non-GI services, and cancelation of scientific conferences. Nevertheless, the impact is not limited to current GI fellows. Current internal medicine residents who are prospective GI fellowship applicants are also severely impacted. GI fellowship is one of the most competitive subspecialty fellowships [4, 5], as evidenced in 2019 when a total of 906 applicants applied for 577 positions [6]. In response to COVID-19, the Electronic Residency Application Service (ERAS) has shifted the timeline for fellowship programs in order to gain access to applications from mid-July to August 12, 2020 [7]. While recent articles have addressed challenges faced by current GI fellows [1, 8], there is limited guidance on how

Niharika Mallepally

rika.mallepally@gmail.com

1 Department of Gastroenterology, University of Southern California, Keck School of Medicine, Los Angeles, CA, USA

2 Division of Gastroenterology and Hepatology, Beth Israel Deaconess Medical Center, Harvard Medical School, 330 Brookline Avenue, Boston, MA 02215, USA

3 Division of Gastroenterology, Massachusetts General Hospital, Harvard Medical School, 55 Fruit Street, Boston, MA 02114, USA

4 Division of Gastroenterology and Hepatology, Johns Hopkins Hospital, Baltimore, 1800 Orleans St, Baltimore, MD 21287, USA

5 Department of Internal Medicine, Division of Gastroenterology and Hepatology, Mayo Clinic, 200 First Street SW, Rochester, MN 55905, USA
GI fellowship applicants should cope with the unprecedented challenges presented by this pandemic. Similarly, fellowship programs and their training directors are also faced with unique challenges since showcasing the strengths of their programs to applicants will be more difficult without the conventional interview process.

We will share the anticipated challenges that will be faced by both applicants and programs for the upcoming fellowship match season, and propose strategies to address these challenges.

\section{Challenges Faced by Applicants}

Prospective GI fellowship applicants will face a unique set of challenges this interview season due to the restrictions imposed by the COVID-19 pandemic that may impact their candidacy (Table 1).

\section{Less Time Spent on GI Elective Rotations}

In spring of 2020, prospective GI fellowship applicants were frequently redeployed in order to provide support to hospital wards and ICU teams, with a consequent reduction in time spent on subspecialty clinical experiences including the development of relationships with GI faculty, with a potential negative impact on the ability to cultivate strong letters of recommendation.

\section{Impact on Research/Scholarly Work}

The pandemic likely slowed the pace of many research projects, especially those that require IRB approval or interactions with patients in clinic. Any elective time that had been initially allocated for research was often converted to frontline hospital coverage in areas hit hardest by COVID19. On the other hand, programs with lower volumes of 


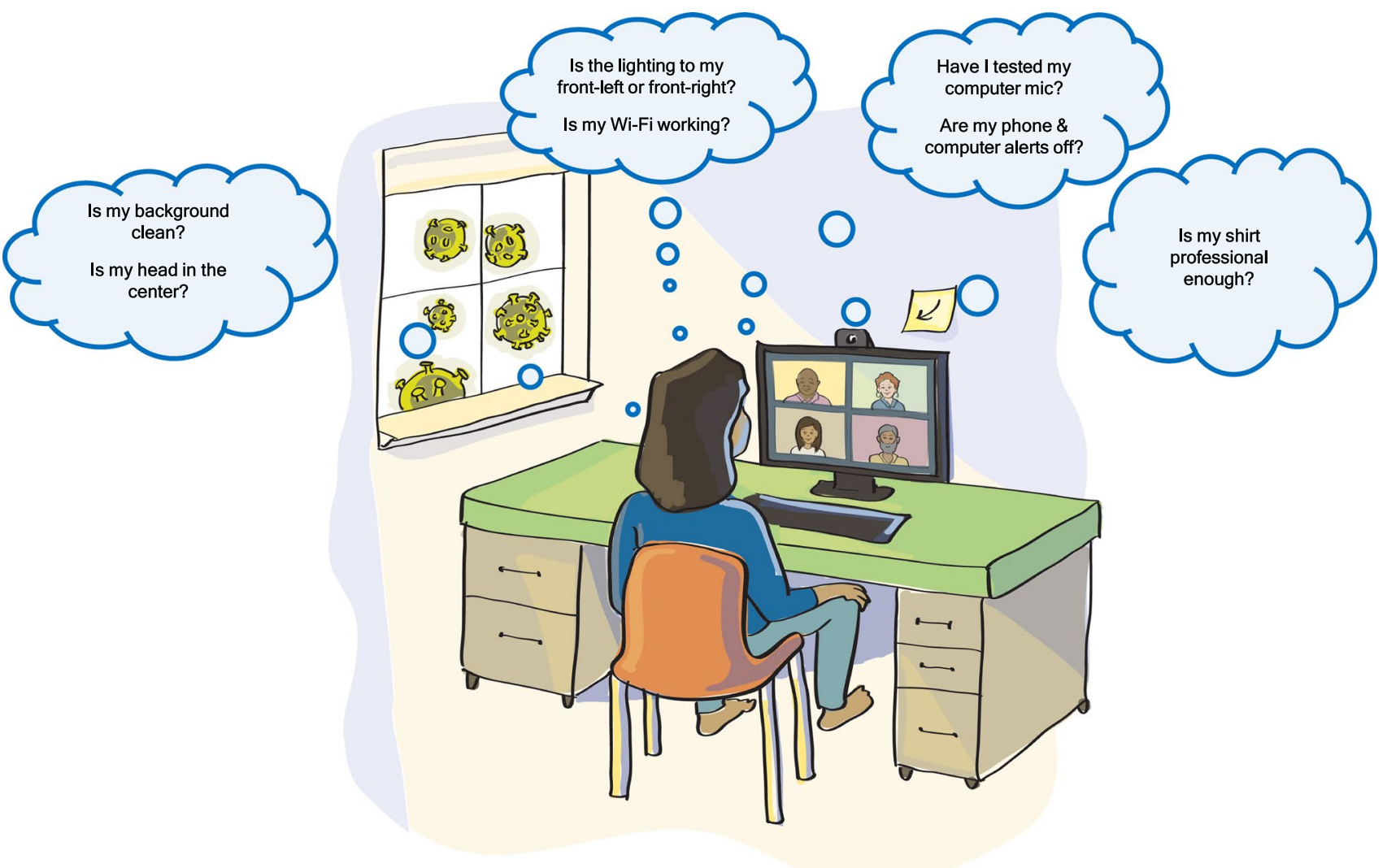

Fig. 1 Preparing for virtual interviews

Table 1 Challenges and solutions for applicants and programs this fellowship interview season

\begin{tabular}{|c|c|c|}
\hline & Challenges & Solutions \\
\hline Applicants & $\begin{array}{l}\text { Less time spent on GI rotations } \\
\text { Less protected research time } \\
\text { Unfamiliarity with virtual interviews } \\
\text { Unsure how programs will consider outside candidates } \\
\text { Unable to assess program or city culture } \\
\text { Inability to build rapport with program faculty and fellows }\end{array}$ & $\begin{array}{l}\text { Participate in online programs hosted by your GI Division (lectures, } \\
\text { conferences) } \\
\text { Ask faculty if abstracts/posters can be converted to manuscripts, focus } \\
\text { on systematic reviews, database research } \\
\text { Mock virtual interviews to prepare } \\
\text { Spend more time on a program's external web site and social media } \\
\text { platforms, if present }\end{array}$ \\
\hline Programs & $\begin{array}{l}\text { Shorter interview season timeline } \\
\text { Creating new interview schedule/format logistics } \\
\text { Training faculty in virtual interviewing } \\
\text { Inability to directly showcase program culture, inter- } \\
\text { faculty interactions } \\
\text { Loss of venues to hold candid conversations between } \\
\text { applicants and current fellows }\end{array}$ & $\begin{array}{l}\text { Train faculty in virtual interviews, stressing a uniform approach to } \\
\text { interviews } \\
\text { Virtual meet and greets with fellows } \\
\text { Robust online and social media presence to share culture } \\
\text { Increase the number of interview slots }\end{array}$ \\
\hline
\end{tabular}

COVID-19 patients may have had more trainees home on "healthy reserve," leading to potential inequalities for trainees from programs most impacted by the pandemic. Furthermore, cancelation of local and national GI conferences has prevented trainees from presenting their research in front of an extramural audience or engaging in valuable face-to-face networking opportunities.

\section{Application Process}

COVID-19 might change application behavior by trainees, with potential apprehension by applicants on leaving their current cities or proximity to family in the middle of the pandemic. Although fellowship for applicants in 
the current cycle will start in July 2021, the uncertainty regarding the residual impact of the pandemic might factor into the number of programs and cities where residents might apply.

\section{Interview Experience}

The loss of in-person interviews will alter how applicants learn about the program's (and city's) culture. Unless virtual methods are developed, applicants will be unable to see how faculty and fellows interact and will not have the ability to network with other applicants "on the trail." It will be much more challenging to evaluate an institution and hospital for compatibility without being able to explore it in person. Furthermore, it will be difficult to know whether a city is livable for an applicant and their family, and they may feel less inclined to move out of cities that are familiar to them.

\section{Challenges Faced by Programs}

Although the angst is undeniable from the applicant perspective, GI fellowship leadership and administration are also facing unique challenges.

\section{Timeline}

Given that the usual date that program directors can review ERAS applications has been delayed from mid-July to midAugust this year, programs will have less time to review applications, interview applicants, and make a rank list than in prior years given other dates, including rank list deadline (mid-November) and match day (early December), have not changed.

\section{Logistics of the Interview Process}

Program leadership will have to determine the logistics of the interview process. Considerations will include determining the number of applicants to interview, time allocation of slots for virtual interviews, interview structure (e.g., panel interviews, 1 on 1, structured interviews, etc.), and how to incorporate a program overview, virtual tour and interactive time with current fellows. Since most faculty may not have training or experience in conducting virtual interviews, the rapid acquisition of the requisite skills is essential.

\section{Program Representation}

Programs use the interview day to showcase their culture, hospitals, faculty, and fellows, given these elements are essential to applicants' decision-making. A major concern for programs is how to remotely share a program's ethos.
Applicants may rely more heavily on a program's external website and social media presence, adding another urgent task for programs if they have not recently dedicated time to updating those platforms.

\section{Applicant Engagement with Current Fellows}

Programs are also concerned about how to facilitate interactions between applicants and current fellows. Fellowship is a team sport mandating collegiality among trainees. Rapport had previously been promoted via interview days and the pre-interview dinner, the latter which has provided a more relaxed environment in which current fellows could communicate program culture to prospective applicants, and applicants could get candid questions answered.

\section{Adapting the Application Process to the Pandemic}

Programs and applicants will need to be creative and utilize all available resources to adapt to the challenges presented by the pandemic. The onus should not fall to program leadership alone, but all faculty (both internal medicine and GI) and peers (both resident and fellows) will need to collectively address the pertinent issues.

\section{For Applicants}

\section{Participation in GI Divisional Activities}

Even though opportunities for direct interactions with local GI faculty may be more limited (as most didactics have been converted to online), interested residents should make efforts to join online lectures and conferences, not only to enhance their education, but also as an opportunity to show interest and engage with GI faculty and fellows.

\section{Scholarly Work/Research}

While cancelation of major GI meetings resulted in fewer opportunities to present research, prospective applicants should embrace social media platforms and share their work in order to engage with peers and thought leaders [9]. Furthermore, residents should work with their research mentors and convert abstracts into manuscripts when possible. While new clinical research project might be challenging to conduct, residents should explore opportunities for scholarly work that would not require need in-person contact (e.g., review articles, meta-analyses, database research, etc.). 


\section{Application Process}

While in-person interviews have historically been a cornerstone in the application process, virtual interviews will incur significant cost savings associated with travel and accommodations. Moreover, since travel time is zero in most cases, trainees will be less apt to use vacation days or days without pay depending on the interview volume. While this can facilitate increased applications to more programs, this could also lead to "application bloat" for program leadership, further straining the tight timeline of the interview season.

\section{Residency Program Support}

Internal medicine residency programs should develop mock interview sessions in order to prepare their trainees who are applying for fellowship using virtual interviews. There are many nuances of virtual interviews of which trainees need to be aware (e.g., professional attire, appropriate background for the video call, dependable cellular or Internet connection, etc.). Residency programs should also ensure that local GI mentors perform periodic check-ins with prospective applicants in order to discuss career goals and answer any questions that will foster the mentor-mentee relationship, make up for lost direct in-person time, and will enable mentors to feel comfortable writing strong letters of recommendations for their mentees.

\section{For Programs}

\section{Train Faculty in Virtual Interview Techniques}

Programs should train faculty members in virtual interview techniques including selecting faculty to interview all applicants in order to establish a cadre of faculty members proficient with virtual interviews that are able to troubleshoot any issues that may arise.

\section{Virtual Meet and Greet}

An integral part of the interview process is allowing applicants to meet current fellows and have a candid and honest conversation about the program. While this has typically been a "meet and greet" during a pre-interview dinner or during a program tour with current GI fellows, it is essential that programs create virtual surrogates for those experiences such as a virtual video call where all the applicants or groups of applicants chat with a few current GI fellows in the absence of any faculty members or program leadership, versus facilitating one-on-one virtual meetings or calls between trainees.

\section{Robust Online and Social Media Presence}

Since programs will not be able to demonstrate their culture in person, the program leadership will need to consider expanding their online and social media presence [10]. Highlighting aspects of the program including wellness, social activities, and relationships among fellows and faculty on websites and social media platforms will be important in order for applicants to understand the program culture.

Some programs, especially that are smaller or more remote, might be at a disadvantage by not having applicants visit in order to showcase their strengths as compared to larger well-established programs. Nevertheless, if online resources are used wisely, programs in smaller cities and newer programs might actually have an improved chance of interviewing applicants since applicants might apply to an increased number of programs due to time and cost savings. Thus, if programs successfully promote themselves online and become more visible to applicants, they may have more favorable outcomes.

\section{Increase the Number of Interview Slots}

Programs should also consider increasing the number of interview slots. Previously, many applicants have had to cancel interviews due to scheduling conflicts and costs associated with travel. In addition to the likelihood of "application bloat," applicants are less likely to cancel interviews due to cost and time savings. Therefore, programs should consider interviewing more applicants to avoid the possibility of going unmatched, balanced against the time demands that each interview will have on faculty.

\section{Virtual Interviews: A Primer}

On the program side, there are a few key points for virtual interviews. First, separating the "program overview" from the individual interviews can be helpful, because the "program overview" session can be more efficiently offered to a larger group of applicants, leaving more time for individual interviews. Second, conducting panel interviews and using a structured interview format can be helpful to avoid the typical challenges of talking over others on Zoom. This also allows for a more standardized and fair approach for selecting applicants during this unique time [11]. Some faculty who have been involved in virtual subspecialty fellowship interviews (author TB) noted that a structured virtual interview approach felt more natural than anticipated. Faculty involved in the interview process should ensure that they 
check their online connection and audio/video ahead of time. Faculty members should ideally conduct these interviews in a quiet setting in order to avoid distractions and offer applicants the sense that they have their undivided attention.

Similarly, many applicants might not be well versed in virtual interviews, even though they might be savvier in using available technologies. The following points are stressed:

1. Choose a quiet room and spend time optimizing your video background, lighting, and camera position ahead of time.

2. A good starting point is to get the camera at eye level and make sure your primary lighting source is coming from your front left or front right and not behind you. We recommend testing out your setup with a colleague on the other line.

3. Wired earbuds may give the best balance of good audio and connection reliability (compared to laptop mic/ speaker or Bluetooth).

4. Turn off other computer alerts (e.g., e-mail, notifications, etc.) during interviews and utilize the "do not disturb" feature.

5. Practice looking into the camera instead of at the interviewer's face on the screen in order to demonstrate good eye contact [12]. If looking down to review or take notes, it will break eye contact, so should be done minimally.

6. Avoid unnecessary disturbances such as distracting backgrounds, fixing hair or glasses, scratching your face, eating or drinking, etc.

7. Practice what you will say and what you want to get across to the interviewer.

\section{Conclusion}

The COVID-19 pandemic will significantly impact the upcoming subspecialty fellowship match. While the changes due to the ongoing pandemic have severely affected current GI fellows, it is important that both residency and fellowship programs prepare residents and prospective fellowship applicants for the upcoming subspecialty match. Programs should pay special attention to how the pandemic might have affected applicants' opportunities regarding scholarly work, electives, and GI experiences. Similarly, applicants should prepare for virtual interviews, and both programs and applicants should be creative and efficient in using all online resources to improve their chances for a successful match. While we recognize that each applicant and program have unique circumstances, we hope this article will identify and address some generalized themes for both applicants and programs.
Acknowledgments The authors acknowledge several residents who chose to remain anonymous for their feedback on issues highlighted in this article. The authors also knowledge Dr Francis Giardiello for this feedback on some issues faced by programs in the upcoming match. The authors also acknowledge Lanessa Miller for preparing Fig. 1.

Funding None with respect to this manuscript.

\section{Compliance with Ethical Standards}

Conflict of interest The authors report no conflict of interest and have no financial disclosures with respect to this manuscript.

\section{References}

1. Keswani RN, Sethi A, Repici A, et al. How to maximize trainee education during the COVID-19 pandemic: perspectives from around the world. Gastroenterology. 2020. https://doi. org/10.1053/j.gastro.2020.05.012.

2. Palchaudhuri S, Gabre J, Prenner S, et al. Adapting a GI fellowship to a pandemic: novel approaches to accommodating a novel virus. Dig Dis Sci. 2020;65:1562-1565.

3. Pawlak KM, Kral J, Khan R, et al. Impact of COVID-19 on endoscopy trainees: an international survey. Gastrointest Endosc. 2020. https://doi.org/10.1016/j.gie.2020.06.010.

4. Duong N, Aby ES, Hathorn KE, et al. How to become a competitive applicant for gastroenterology fellowship: tips and tricks for success part II. Dig Dis Sci. 2020. (Epub ahead of print). https:// doi.org/10.1007/s10620-020-06293-4.

5. Duong N, Aby ES, Hathorn KE, et al. How to become a competitive applicant for gastroenterology fellowship: tips and tricks for success part I. Dig Dis Sci. 2020. (Epub ahead of print). https:// doi.org/10.1007/s10620-020-06292-5.

6. Match Results Statistics. https://mk0nrmp3oyqui6wqfm.kinst acdn.com/wp-content/uploads/2019/12/415_MRS.pdf. Accessed 30 May 2020.

7. Coronavirus (COVID-19) and ERAS. https://students-residents. aamc.org/applying-residency/article/coronavirus-covid-19-anderas/. Accessed 30 May 2020.

8. Shah R, Satyavada S, Ismail M, et al. COVID-19 pandemic through the lens of a gastroenterology fellow: looking for the silver lining. Gastrointest Endosc. 2020. https://doi.org/10.1016/j. gie.2020.03.3852.

9. Bilal M, Taleban S, Riegler J, et al. The Do's and Don'ts of social media: a guide for gastroenterologists. Am J Gastroenterol. 2019; $114: 375-376$.

10. Gabrielson AT, Kohn JR, Sparks HT, et al. Proposed changes to the 2021 residency application process in the wake of COVID-19. Acad Med. 2020. https://doi.org/10.1097/ACM.000000000000352 0 .

11. Patrick LE, Altmaier EM, Kuperman S, et al. A structured interview for medical school admission, phase 1: initial procedures and results. Acad Med. 2001;76:66-71.

12. Jones RE, Abdelfattah KR. Virtual interviews in the era of COVID-19: a primer for applicants. J Surg Educ. 2020;77:733734. https://doi.org/10.1016/j.jsurg.2020.03.020.

Publisher's Note Springer Nature remains neutral with regard to jurisdictional claims in published maps and institutional affiliations. 